\title{
Negative refraction due to discrete plasmon diffraction
}

\author{
Arian Kriesch ${ }^{1,2}$, Ho Wai Howard Lee ${ }^{2}$, Daniel Ploss ${ }^{1}$, Stanley P. Burgos ${ }^{2}$, Hannes Pfeifer ${ }^{1}$, Jakob Naeger ${ }^{1}$, \\ Harry A. Atwater ${ }^{2}$ and UIf Peschel ${ }^{1}$ \\ ${ }^{1}$ Inst. of Optics, Information and Photonics \& Graduate School in Advanced Optical Technologies, University Erlangen-Nuremberg, Germany \\ ${ }^{2}$ Thomas J. Watson Institute of Applied Physics, California Institute of Technology, Pasadena, USA \\ arian.kriesch@mpl.mpg.de
}

\begin{abstract}
We experimentally demonstrate spectrally broad $\left(\lambda_{0}=1200-1800 \mathrm{~nm}\right)$ in-plane negative diffraction of SPPs in an array of plasmonic channel waveguides with negative mutual coupling resulting in negative refraction on the array's interface and refocusing in an adjacent metal layer. OCIS codes: (240.6680) Surface Plasmons; (250.5403) Plasmonics; (160.3918) Metamaterials
\end{abstract}

Negative refraction, first investigated by V. Veselago [1], has attracted great interest since its reinvention by J. Pendry [2]. With the recent advent of nanofabrication techniques first realizations with optical metamaterials have been reported [3], with the particularly exciting possible application of superlensing. At the same time, discrete diffraction in artificially anisotropic optical materials attracted its own popularity for its system simplicity, designability and interesting linear and nonlinear properties, taking benefit from the rush of industrial development of dielectric photonics [4]. However, most of these applications are narrow-band and usually it is not possible to directly observe the light propagation or to apply these materials in an accessible planar geometry, although this would open insights into negative refraction and applications in planar nanophotonics.
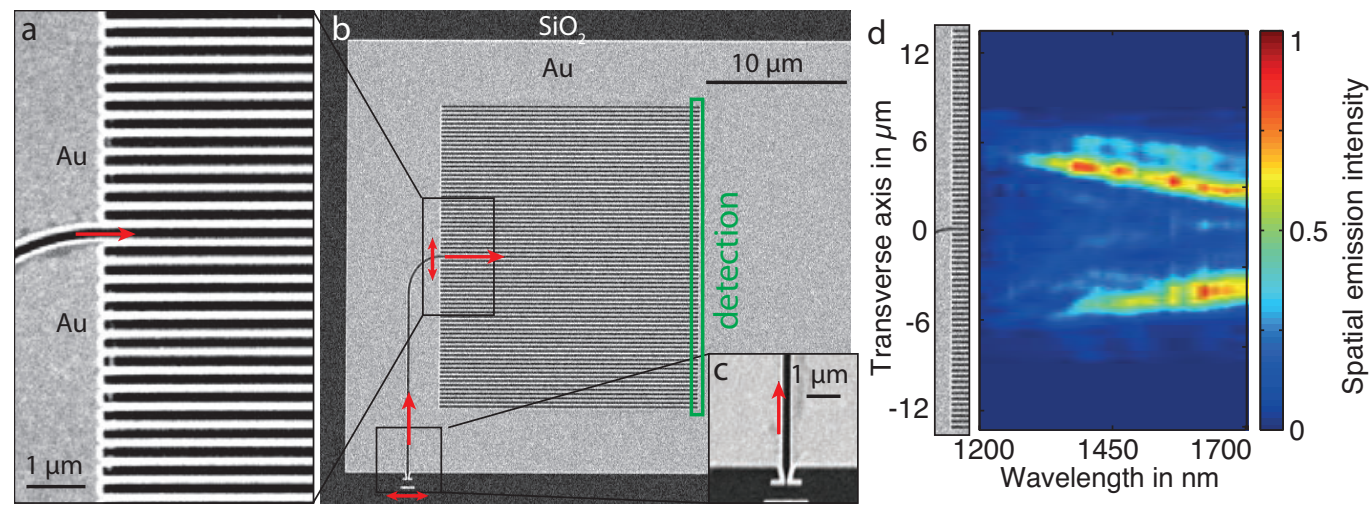

Figure 1 (a) A plasmonic array (b) connected with an SPP channel waveguide and excited via (c) a Yagi-Uda nanoantenna (d) shows $2 \mathrm{D}$ discrete diffraction with a strong spectral dispersion and negative coupling.

Here we transfer the concept of discrete diffraction from arrays of dielectric waveguides [5] to arrays (nanoscale confinement, ca. $300 \mathrm{~nm} \approx \lambda_{0} / 5$ ) of coupled plasmonic channel waveguides (pitch $\Gamma \approx 370 \mathrm{~nm}$ ).

We demonstrate for the first time intrinsic negative coupling in this array in the discrete diffraction process that we exploit to achieve 2D negative refraction while we experimentally directly monitor the wave propagation with farfield and near-field measurements. The negative coupling leads to an inverted wavefront curvature that causes negative diffraction that we observe when the bound modes from the array are converted to surface plasmon polaritons on a connected plane metal surface.

We excite a single gap mode of the array by connecting a channel SPP waveguide (Fig. 1a,b) that is excited with a highly focused laser beam with a continuously scanned wavelength via acousto-optical tunable filters $\left(\lambda_{0}=1200-\right.$ $1800 \mathrm{~nm}$ ) via a connected Yagi-Uda nanoantenna (Fig. 1b and c) with a power conversion efficiency of $15 \%$ and a typical propagation length of $30 \mu \mathrm{m}$ [6]. When this feed waveguide enters the array the mode of this first waveguide evanescently couples to the modes of the adjacent waveguides.

This causes discrete diffraction inside the array, which acts as a highly anisotropic 2D metamaterial. For a single waveguide coupler with this design we demonstrated a shortest achievable coupling length of only $2.45 \mu \mathrm{m}$, compared to typically millimeters in dielectric photonics and with a high spectral dispersion that is governed by the dispersion of the metal filaments between the waveguides [6]. Here we observe that the field distribution inside the array causes a laterally diverging intensity distribution with two distinct maxima at the diverging outer bounds at the distance $W_{\text {a }}$ from the excited center waveguide (Fig. 1d), which is in full accordance with the theoretical description of discrete diffraction for dielectric waveguide arrays [4,5]. 
We conducted full 3D FDTD calculations and investigated the spectral dispersion over a broad wavelength range to calculate the full band diagram of the investigated modes (Fig. 2e). Discrete diffraction theory predicts a wave vector $\left(\boldsymbol{k}=\beta \mathbf{e}_{\|}+\kappa \mathbf{e}_{\perp}\right)$ dependence of $\beta=\beta_{0}+2 c \cos (\kappa \Gamma)$, where negative diffraction is characterized by a negative coupling constant $c$. Indeed, we observe a negative coupling constant in our calculations (Fig. 2e, white highlight) over an extremely broad wavelength range. In the corresponding field distributions we see an inverse coupling phase between adjacent SPP waveguides caused by the polarization building up on the metal ridge, that is a fundamental difference to the coupling effect in dielectric waveguides. This negative coupling constant is the basic prerequisite to achieve negative refraction.

From broadband measurements of the intensity distribution at the array end (Fig. 1d) we determined the coupling constant $c=W_{\mathrm{a}} /(2 L \Gamma) \approx 0.34 \mu \mathrm{m}^{-1}$, resulting in an extremely large anisotropy of the effective refractive index $\Delta n_{\mathrm{eff}}=2 \lambda_{0} c / \pi \approx 3.14$. We also determined the spectral dependence of the wave spreading $W_{\mathrm{a}}(\lambda)$ (Fig. $\left.1 \mathrm{~d}\right)$. It is opposite to the known dispersion in dielectric arrays, consistently indicating a negative coupling process.

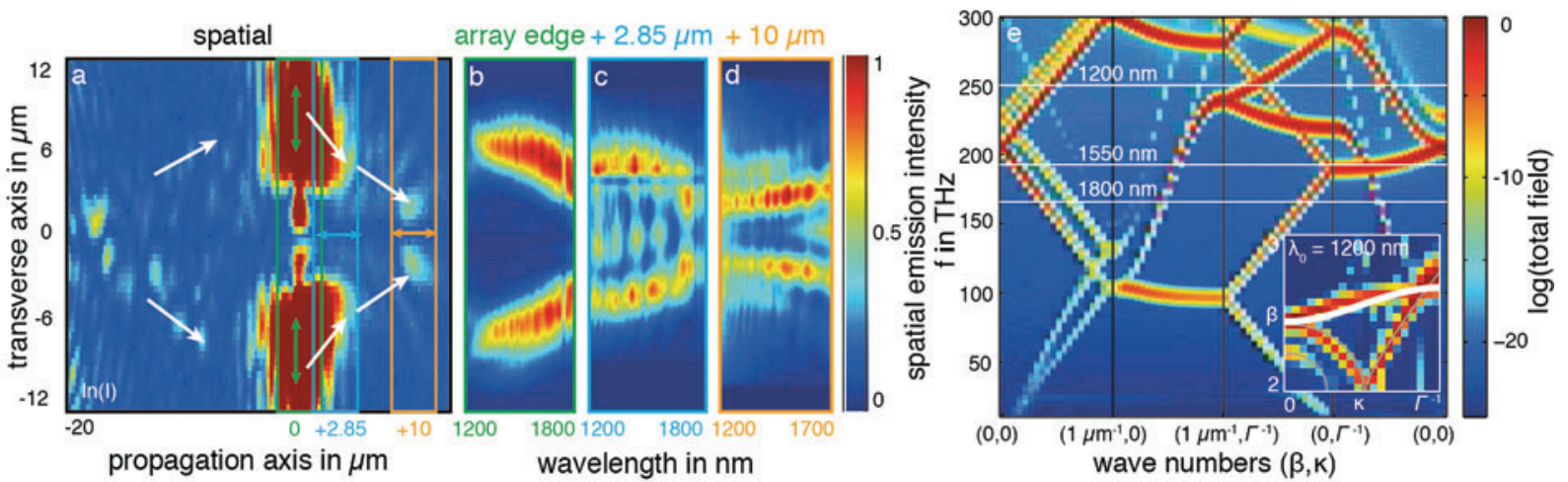

Figure 2(a) Discrete diffraction in the array shapes two diverging maxima. At the array end $(0 \mu \mathrm{m})$ a fraction of the bound modes is scattered into free-space (b), some is converted into propagating SPPs. Negative refraction at this interface causes the SPPs to focus ( $c$ and d). (e) Band structure calculations reveal the (- cos shaped) negative refraction band (white highlight for $\lambda_{0}=1200 \mathrm{~nm}$ ) in the $\beta, \kappa$ space (grey: backfolded isotropic modes), over a broad spectral range.

With high-sensitivity NIR microscopy we directly observed the wave propagation inside a $25 \mu \mathrm{m}$ long array (Fig. 2a). In this case we coupled a focused laser beam directly into the array edge and achieved the same coupling characteristics as with the antenna excitation. Due to the negative coupling, the array waves accumulate phase during their propagation that leads to an opposite phase front curvature than it is achievable with dielectric arrays. Where the array ends, part of the bound modes is converted to far-field light that we directly monitored (b) and part of it is converted into a bound surface plasmon mode that clearly focuses (Fig. 2 b,c vs. d) while it propagates on the plane metal. It is subsequently monitored after $2.85 \mu \mathrm{m}(\mathrm{c})$ and $10 \mu \mathrm{m}(\mathrm{d})$, each mode with its specific polarization (Fig. 2a green vs. blue and orange). The spectral measurement of the propagating waves (Fig. 2b,c and d) shows the dispersion of discrete diffraction (Fig. 2b) and of the subsequent refocusing and spreading of the SPP (Fig. 2c and d). It confirms negative diffraction for the array in a broad spectral range and shows that its dispersion is completely different from that of SPPs.

To summarize, we have demonstrated that modes of metallic arrays are subject to discrete diffraction with strong negative coupling resulting in a strong anisotropy of the effective index. We furthermore directly observed focusing of a planar SPP, emerging from the array, which clearly indicates 2D negative plasmon refraction and is the prerequisite for 2D superresolution imaging.

\section{References}

[1] V. G. Veselago, "The electrodynamics of substances with simultaneously negative values of $\mu$ and E," Sov. Phys. Uspekhi 10, 509-514 (1968).

[2] J. B. Pendry, "Negative Refraction Makes a Perfect Lens," Phys. Rev. Lett. 85, 3966-3969 (2000).

[3] V. M. Shalaev, "Optical negative-index metamaterials," Nat. Photonics 1, 41-48 (2007).

[4] D. N. Christodoulides, F. Lederer, and Y. Silberberg, "Discretizing light behaviour in linear and nonlinear waveguide lattices.," Nature 424, $817-823$ (2003).

[5] U. Peschel, T. Pertsch, and F. Lederer, “Optical Bloch oscillations in waveguide arrays.," Opt. Lett. 23, 1701-1703 (1998).

[6] A. Kriesch, S. P. Burgos, D. Ploss, H. Pfeifer, H. A. Atwater, and U. Peschel, "Functional plasmonic nanocircuits with low insertion and propagation losses.," Nano Lett. 13, 4539-4545 (2013). 